\title{
OH/IR STARS AS SIGNPOSTS FOR ANCIENT STARBURST ACTIVITY IN THE GALACTIC CENTER
}

\author{
L.O. SJOUWERMAN
}

Onsala Space Observatory and Sterrewacht Leiden

H.J. HABING

Sterrewacht Leiden

H.J. VAN LANGEVELDE

Joint Institute for VLBI in Europe

AND

M. LINDQVIST AND A. WINNBERG

Onsala Space Observatory

We have surveyed the Galactic center (GC) for OH/IR stars, evolved late-type stars of different masses - and thus ages - in a short-lived stage of heavy mass-loss. By observing the $1612 \mathrm{MHz} \mathrm{OH}$ masers generated in their circumstellar shells, it is found that a considerable fraction of these $\mathrm{OH} / \mathrm{IR}$ stars has the same high-valued shell expansion velocity of $19 \mathrm{~km} \mathrm{~s}^{-1}$.

The shell expansion velocity is related to the opacity (metallicity) of the circumstellar material and the stellar luminosity (Habing et al. 1994, A\&A 286, 523). Combining several observable properties of these objects, we argue that the GC has undergone a distinct epoch of star formation more than a Giga-year ago, in which a large number of stars have formed.

¿From the observations it seems that in the GC three different groups of stars can be traced: 1) an overall old, dynamically relaxed low-mass, low expansion velocity bulge-like population; 2) a dynamically tight group of relatively young stars formed from continuously infalling clouds; and 3) a shrinking population of (now) low-mass stars, formed from a rapidly rotating molecular cloud that suddenly - more than a Gyr ago-transformed into stars at a high rate.

We hereby combine, and support the views of Lindqvist et al. (1992 A\&A 259, 118), and both the view of Sevenster et al. (1995 A\&A 299, 689) 
and the view of Serabyn \& Morris (1996 Nature 382,602) that contradicts the view of Sevenster (1995).

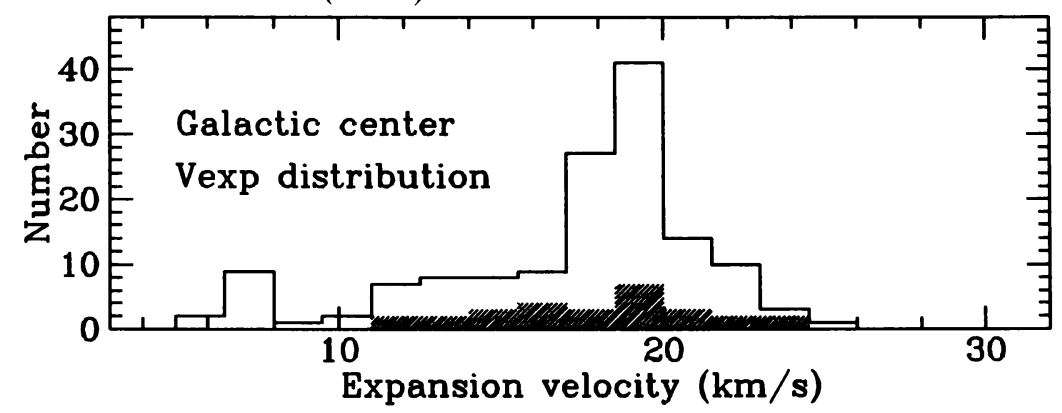

We present a histogram of a new sample of $\mathrm{OH} / \mathrm{IR}$ stars in the GC, (Sjouwerman et al. $1997 \mathrm{~A} \& \mathrm{AS}$ in press), ordered by $v_{\exp }$. The GC $v_{\exp }$ distribution is much more peaked than any other sample known, (eg. OH/IR stars in the Galactic bulge and disk; Sevenster et al. 1997a A\&AS 122, 79, 1997b A\&AS 124, 509), and has a relatively high mean value of $19 \mathrm{~km} \mathrm{~s}^{-1}$. The very narrow $v_{\text {exp }}$ distribution indicates that many of the " $19 \mathrm{~km} \mathrm{~s}^{-1}$ " $\mathrm{OH} / \mathrm{IR}$ stars have the same age.

The strongest argument that they were formed in a short period is based on dynamical grounds. The difference in velocity dispersion and sky distribution for low (large dispersion) and high (small dispersion) $v_{\exp }$ stars is evidence that the general group of high $v_{\exp }$ stars - which includes our " $19 \mathrm{~km} \mathrm{~s}^{-1}$ " stars - have a shorter dynamical evolution; they were formed later (Lindqvist et al. 1992 A\&A 259, 118; Sevenster et al. 1995 A\&A 299, 689 ). The old age ( $>1 \mathrm{Gyr}$, see below), the strong rotation in the plane, and the fact that the " $19 \mathrm{~km} \mathrm{~s}^{-1}$ " $\mathrm{OH} / \mathrm{IR}$ stars have a velocity dispersion similar to the highest $v_{\exp }\left(>20 \mathrm{~km} \mathrm{~s}^{-1}\right)$ stars, indicate that many of the "19 $\mathrm{km} \mathrm{s}^{-1}$ " $\mathrm{OH} / \mathrm{IR}$ stars originate from a single cloud circling the center.

Bolometric luminosities for GC OH/IR stars (Blommaert et al. 1997, $\mathrm{A} \& \mathrm{~A}$ in press) are not representative for the whole sample (eg. in $v_{\text {exp }}$, shown as the shaded $v_{\exp }$ distribution). We therefore may hypothesize that many of the " $19 \mathrm{~km} \mathrm{~s}^{-1}$ " stars have roughly the same luminosity $\left(\mathrm{M}_{\mathrm{bol}} \approx\right.$ -4.5 ). Models then predict a mass of less than $2 \mathrm{M}_{\odot}$ and an age $>1$ Gyr. As the stars were formed in a relatively isolated period, the " $19 \mathrm{~km} \mathrm{~s}^{-1}$ " stars are the most massive representatives from that period. Because the $\mathrm{OH} / \mathrm{IR}$ phase is so short $\left(10^{5} \mathrm{yr}\right)$, this means that many of the " $19 \mathrm{~km} \mathrm{~s}^{-1}$ " stars have the same mass (and age), ie. in a very narrow range and independent of the length of the formation because they formed so long ago.

Given the lower limits on the number of stars formed (a few $10^{7}$ over all masses), the mass of the original rotating cloud (a few times $10^{8} \mathrm{M}_{\odot}$ ), and the luminosity produced in the nucleus during this event $\left(\sim 10^{10} \mathrm{~L}_{\odot}\right)$, the Galaxy would probably have been classified as a starburst galaxy. 